\title{
OBSTETRICS IN GENERAL PRACTICE
}

\section{The Puerperium}

\author{
G. GORDON LENNON,* CH.M., F.R.C.O.G., M.M.S.A. ; MICHAEL LENNARD, † M.B., CH.B.
}

The puerperium may be defined as that period which extends from the delivery of the baby and placenta until the return of the genitalia to the non-pregnant state. This occupies from six to eight weeks.

\section{The Uterus}

\section{Physiology}

The physiology of the puerperium is concerned with retrogressive changes, both local and general, and progressive changes in the breasts. The retrogressive changes of the genitalia are grouped under the general term involution. The greatest degree of involution is shown by the uterus, which immediately after delivery of the placenta weighs in the region of $2 \mathrm{lb} .(0.9 \mathrm{~kg}$.). At the end of two months it will have involuted to a weight of about $2 \mathrm{oz}$. (56 g.). After delivery the fundus of the uterus is at the umbilicus and 10 days later it is at or below the level of the symphysis pubis. The ligaments of the immediately puerperal uterus are so lax that the uterus can be pushed about easily, especially so by urine in the bladder. At any examination, therefore, of the uterus at this time the bladder must be empty. The contractions of the uterus after delivery for a few days may be quite severe and painful and are called " afterpains." Relief of these " after-pains" may be obtained by prescribing sedatives such as tabs. sodium amytal gr. 3 (0.2 g.), or tabs. codeine phosphate gr. $\frac{1}{2}(32 \mathrm{mg}$.). Hormone therapy should be avoided because of possible interference with involution and breast changes.

The cervix after delivery hangs like a soft cuff, bruised and oedematous. Within 48 hours the cervix forms, shortens, and becomes harder. By the 12th day one finger can reach the internal os but cannot go through it. By the 4th week the external os is a small transverse slit. The interior of the uterus after the placenta is out is one large wound with immense blood sinuses at the placental site. These sinuses contain superficial thrombi. Bleeding has been controlled by the contraction and retraction of the interlacing muscle fibres. Soon the epithelial cells from the stumps of the glands lying along the muscle fibres proliferate and grow out on the surface, constituting a new endometrium. The discharge from the uterine cavity, the lochia, is at first blood (lochia rubra). Gradually the discharge gets less in amount and less bloody (lochia alba) till by the end of a fortnight there is only a slight muco-serous discharge.

The abdominal wall, the vagina, the uterine ligaments, and the supporting structures in the pelvis also involute gradually.

\section{The Breasts}

Successful and progressive involution is aided by the stimulation of breast activity. In two to three days the breasts

* Professor of Obstetrics and Gynaecology, University of Bris:ol.

t Gezeral Practitioner. grow heavier and fuller, and the superficial veins become prominent. In primiparae the milk comes into the breasts quite stormily-" shooting in "-about the third day. Until then colostrum is all that has been formed.

Colostrum is a fluid only slightly cloudy and sticky, and under the microscope is seen to be made up of fat globules, a watery fluid, and colostrum corpuscles; these corpuscles are round, ovoid, or stellate cells, sometimes showing amoeboid movement and having one to three nuclei. They contain fat globules. Colostrum contains very little, if any, casein, but contains nearly $15 \%$ lactalbumin and lactoglobulin with much fat.

Human milk is an opaque bluish-white fluid with a sweetish taste. It contains $88 \%$ water, $2 \%$ protein, $4 \%$ fat, and $6 \%$ sugar. The iron content is $1.7 \mathrm{mg}$. per litre.

\section{First Day}

\section{Examination During the Puerperium}

It is essential that the woman should have a good sleep after she has been washed up by the midwife after delivery. Swabbing of the vulva (" hibitane" (chlorhexidine) 10\%) will be carried out once in the morning and once in the evening. The patient will be allowed up to wash. Her temperature and blood-pressure will be recorded morning and evening. The baby will be put to the breast six to eight hours after delivery. The patient will be instructed to wash her hands and breasts with soapy water before feeding the baby, and after each feed she will put lanolin on the nipples. (Throughout the puerperium it is essential for the woman to have normal meals with protein and green vegetables and plenty of fluids.)

\section{Second Day}

Vulval swabbing will be carried out in the morning only and that same evening the patient will be allowed a bath in which there is $2 \%$ hexachlorophene. She should powder the navel and skin flexures with hexachlorophene powder. After micturating she should use a sterile towel pad. Blood for follow-up haemoglobin estimation should be taken.

\section{Third, Fourth, and Fifth Days}

The milk now comes into the breasts. The temperature will be taken in the evening only. The breasts may become engorged. If so, they should be bathed in hot water and gentle massage performed by the midwife before the baby is put to the breasts. If the breasts are so engorged that they are causing constitutional upset (slight pyrexia, a complaint of pain and depression) then the breasts should be bound firmly and the patient given $0.05 \mathrm{mg}$. of ethinyl oestradiol.

Perineal sutures may be removed on the fifth day. 


\section{Tenth-day Examination}

In the normal case a 10th-day examination should be carried out by the doctor. This includes blood-pressure, urine tests, examination of breasts and nipples, assessment of abdominal muscle tone, examination of the legs to exclude thrombosis, and a digital pelvic examination to assess involution, the healing of perineal lacerations, and the state of the lochia. The baby should also be examined and its weight recorded. The doctor should instruct the patient not to have intercourse until the end of one month after delivery. He should also discuss with her the importance of further post-natal examinations at the end of six weeks and three months. In many cases the possibility of contraception should be tackled and the patient referred to a clinic for this if the doctor is unwilling to carry this out himself.

\section{Sixth-week Examination}

This is exactly as at the 10th-day examination except that there should be no lochia. Any erosion of the cervix is noted.

\section{Third-month Examination}

This should be the final examination and includes the procedures adopted for the previous post-natal examination. Erosion of the cervix should be cauterized if present, after a vaginal cytology smear has been taken. A final haemoglobin estimation should be done.

\section{Post-natal Exercises}

These exercises should be done six times each hour. 1st Day

1. Lying with knees crooked: (a) Breathe in gently, expand the abdominal wall. Breathe out and pull in hard. (b) Breathe in and expand ribs sideways. Breathe out as deeply as possible.

2. For the pelvic floor muscles. Lying with ankles crossed, pull up inside, as if trying not to pass urine.

The following exercises should be done six times twice daily. 3rd Day

3. Add the following: Lying on back with knees crooked up: (a) Pull in the lower part of the abdomen and tighten the buttocks and tuck in the "tail," trying to pull the front of the pelvis up towards the chest. (b) Relax and allow the spine to hollow.

4. (a) Stretch out straight, leg towards the end of the bed. (b) Draw it up into the body (keeping it straight) and make it shorter; change legs and repeat.

5. Rotate knees from side to side.

5th Day

6. Add the following: Kneel on all fours, then hump and hollow.

7. (a) Turning to look at right hip then left hip alternately. (b) Stand with back against wall and pull up so that the body is in contact with the wall. Keep this position up when walking and standing.

Practise the exercises daily until three months after the baby's birth and remember to hold the body in the correct position when standing and sitting.

\section{Puerperal Pyrexia}

Notifiable puerperal pyrexia is a temperature of $100.4^{\circ} \mathrm{F}$. ( $38^{\circ}$ C.) on one occasion within 14 days of childbirth.

The midwife should refer to medical aid for (i) temperature of $99.4^{\circ} \mathrm{F}$. ( $37.4^{\circ} \mathrm{C}$.) on three successive occasions. (ii) Continuing rise in pulse-rate without pyrexia. Pyrexia may be due to extragenital or genital infection, but any pyrexia is considered due to genital infection until it is proved otherwise.

\begin{tabular}{|c|c|}
\hline Extragenital infections & Genital infections \\
\hline $\begin{array}{l}\text { Throat } \\
\text { Breasts } \\
\text { Urinary }\end{array}$ & $\begin{array}{l}\text { Infected lacerations } \\
\text { Intrauterine } \\
\text { Pelvic cellulitis }\end{array}$ \\
\hline Thrombotic $\left\{\begin{array}{l}\text { Thrombophlebitis } \\
\text { Phlegmasia alba } \\
\text { dolens ("white leg") }\end{array}\right.$ & $\begin{array}{l}\text { Pelvic peritonitis } \\
\text { (Bilateral salpingo-oophoritis) } \\
\text { Infected ovarian cyst }\end{array}$ \\
\hline
\end{tabular}

\section{History}

The patient may have no symptoms. She may complain of undue fullness of the breasts (third or fourth day). She may have a rigor. She may complain of discomfort and/or pain almost anywhere. The history of the confinement is very important especially with regard to interference and trauma. Was the patient catheterized? Was she given an anaesthetic? If so, did she vomit during induction? Was there extensive blood loss? Were placenta and membranes delivered completely? Was there pyrexia during labour? Have the lochia been normal? Is there pus and offensive discharge ? Have the lochia suddenly stopped?

\section{Examination}

Take the blood-pressure, pulse-rate, and temperature.

The throat, chest, and breasts must be examined carefully. Abdomen. The uterus may be unduly large and tender (intrauterine infection). The patient may be very tender and rigid on one or both sides of the uterus (peritonitis), and crepitus may be felt (Clostridium welchii).

There may be swelling apart from, the uterus-for example, an ovarian cyst, which is tender.

Per vaginam. The vulval pad should be examined. Is there pus? Is it offensive? The perineum may be oedematous with stitches cutting in.

Before any digital examination is made, a sterile speculum should be passed and a high vaginal swab taken to send to the laboratory. The infecting organism is usually of the following :

1. Haemolytic streptococcus.

2. Staphylococcus aureus.

3. Anaerobic streptococcus.

4. Coliform bacilli.

5. Cl. welchii.

Other organisms may be grown but are of minor importance. The speculum may reveal a piece of placenta or membrane bulging through the open cervix; a tear of the cervix may be seen.

Vaginal examination is completed by digital palpation of the pelvis, using a sterile glove and "dettol" or " hibitane" cream. The size of the cervix should be noted. The uterus may be enlarged more than it should be, and it may be tender. The possibility of a haematoma or ovarian cyst on either side of the uterus should be considered.

\section{Other Investigations}

1. A mid-stream specimen of urine, or catheter specimen, should be sent to the laboratory for detection of urinary infection.

2. X-rays: (a) Chest: In cases of respiratory infection, and suspected pulmonary embolus, this will be necessary. (b) Pelvis and lower abdomen: This may be helpful if an ovarian cyst is suspected. Bone or a tooth in a dermoid cyst may be seen. 
3. The patient's legs must be carefully examined for phlebothrombosis, even when there is no complaint of pain referred to them. There may be oedema of one foot or leg. Redness and tenderness over a thrombosed vein may be seen and felt. Homan's sign may be positive (pain in the vein elicited by sharp dorsiflexion of the foot); it is positive in about half the cases (see later).

4. During the rigor blood is removed for blood-culture (septicaemia).

5. Blood may be taken and allowed to clot, and a pink staining of the serum is seen. This indicates haemolysis in severe infection ( $\mathrm{Cl}$. welchii).

6. White blood-cell count.

7. Colpotomy: The patient with pelvic peritonitis (bilateral salpingo-oophoritis) may develop a pelvic abscess. The symptoms of this are lower abdominal pain, rectal tenesmus, diarrhoea, and swinging pyrexia. It may rupture spontaneously into the rectum with great relief. Before rupture, it may be diagnosed on vaginal examination. Apart from extreme tenderness in the uterorectal pouch, a cystic swelling will be palpated. It is important to remember that the uterorectal pouch (pouch of Douglas) is an immediate relation of the upper third of the posterior vaginal wall. The pus will cause this area to bulge, so that characteristically on palpation the fingers in the vagina will be immediately guided on to the posterior lip of the cervix-that is, the posterior fornix is obliterated. A needle inserted in this region (colpotomy) will allow the pus to be removed for diagnostic purposes.

It is appreciated that as far as the general practitioner is concerned he will have called in a consultant colleague on suspecting respiratory infection or pelvic peritonitis, or thrombosis.

\section{Urinary Complications}

1. Inability to pass urine. (The subject of anuria following childbirth is not within the scope of this article, but nevertheless it is important that the general practitioner should understand that anuria can occur, and that he should have consultant advice about it.)

The patient may have passed urine on one occasion previously, but it proved so painful due to vulval lacerations that she is now afraid of the dysuria and therefore unwilling to micturate. Apart from these considerations she may find that although she has an intense desire to pass urine she cannot. This last symptom is usually due to psychological upset, and it is therefore important to sit down and discuss the matter with the patient. The midwife will already have tried the usual tricks such as running the tap water. In the end it may be necessary to pass a catheter. This should be done with the utmost aseptic precautions, and preferably $2 \mathrm{oz}$. $(57 \mathrm{ml}$.) of $1: 1,000$ watery "hibitane" should be passed through the catheter when the urine has been let off. This will prevent subsequent urinary infection.

2. Urinary infection. Even although a patient has not been catheterized she may develop acute infection in the puerperium. It will be recognized because of pyrexia $\left(101^{\circ} \mathrm{F} .-38.3^{\circ} \mathrm{C}\right.$.), dysuria, perhaps even pain in loins (particularly on the right side), and constitutional upset such as headache and vomiting. It will best be treated by fluids to drink and sulphonamides. A specimen of urine should be obtained as a mid-stream specimen, or even by catheterization, and sent for identification of the infecting organism and sensitivity to the chemotherapy used. On completing the course of drug prescribed, another specimen should be sent 24 hours after suspension of treatment to ensure that the infection has been overcome. These considerations are extremely important when one considers that of all women coming into an obstetric department 7 to $8 \%$ have asymptomatic urinary infection.

\section{Sleep}

It is very important that the woman should sleep, since she has a busy life ahead of her, with her baby and herself to look after ; sedatives may therefore be given. Sleep is also an important consideration in the prevention of psychiatric disorders of the puerperium.

\section{Psychiatric Illness of the Puerperium}

Psychoneurosis appearing at the puerperium is rare and need not be discussed.

Psychosis, which occurs in about $0.12 \%$ of confinements, is a grave complication as it carries risk to mother and child, causes an interruption in the maternal relationships, and in most cases does not clear up for weeks or months.

Nowadays depression is the form most frequently seen; the once-common delirium, due to exhaustion, infection, or toxaemia, has happily almost disappeared as a result of modern obstetric technique and the use of antibiotics. What was called "puerperal mania" was probably delirium. There is no such thing as milk fever or lactational psychosis. The idea that feeding or failing to breast-feed an infant caused psychosis has no foundation and milk fever was probably a toxic reaction to breast infection.

The incidence of forms of puerperal psychosis is as follows: $50 \%$ affective illness-that is, forms of depression and mania; $20 \%$ schizophrenia ; the remainder percentage is made up of delirium, confusion, and mixed schizo-affective disorders.

Puerperal psychosis appears soon after delivery and the peak is about the 14th day. In the majority of cases it cannot be foreseen and, especially with depression, the mother may have been well and optimistic right through pregnancy. With the exception of schizophrenia, all patients will recover, provided intercurrent illness and suicide have been avoided.

Delirium due to exhaustion and infection usually clears up in a few days and may be treated in the maternity hospital. The other illnesses require admission to a psychiatric unit without delay.

Indications that a puerperal psychosis is developing are restlessness or listlessness, loss of sleep, unexpected emotional changes, irrational talk, morbid ideas, and hostility towards the child. Screaming, violent behaviour, and restiveness are grave signs and usually indicate schizophrenic excitement or mania. Misinterpretation of the environment and distortion of what the patient sees suggest delirium.

\section{Breasts}

At any time the breasts may become infected, which will be evidenced by constitutional upset and local engorgement with pain. The patient should be instructed to report at once in the event of tenderness, redness, or a lump in the breast. Early treatment with penicillin (inj. penicillin 500,000 U.) and ice packs will often give dramatic relief and prevent formation of pus. Normal breast-feeding should continue. Ice may be difficult to obtain in domiciliary practice: ice-cream (in a "polythene" bag) makes an efficient substitute and is usually readily available in any district. Certainly if the infecting organism is sensitive it should respond quickly: once pus has developed it must be let out, but breast-feeding will probably then have to be discontinued. Efficient prenatal breast preparation may help to prevent cracked nipples, the normal source of entry of infection. In this connexion, prevention of breast infection is all-important ; it should be carried nut bv treating the baby's flexures and umbilicus with hexachlorophene soap and " $\mathrm{zac"} \mathrm{(hexachlorophene} \mathrm{1 \% )} \mathrm{powder.} \mathrm{This} \mathrm{inhibits} \mathrm{the}$ rapid normal invasion of staphylococci in these areas. 
In addition to the engorgement of the breasts previously mentioned it may be necessary for the patient to inhibit breastfeeding, and the milk therefore has to be suppressed. Fluids should be limited, the breasts bound up tightly, and the patient given stilboestrol for five days; $5 \mathrm{mg}$. three times a day on the first day, $4 \mathrm{mg}$. on the second, $3 \mathrm{mg}$. on the third, $2 \mathrm{mg}$. on the fourth, and $1 \mathrm{mg}$. on the fifth day.

\section{Bleeding}

The patient may have a reactionary haemorrhage on the first day, perhaps due to bleeding from a laceration. This is especially likely when the uterus is palpated and felt to be hard. This is rather a matter for the Emergency Obstetric Service, which is dealt with in a separate article. We have known the bleeding to be so severe because of an incompetently stitched perineum that the patient had to be admitted to hospital after being visited by the Emergency Obstetric Service. Should the bleeding be accompanied by a soft, large uterus, there will be no doubt that there is some retention of fragments of the placenta in the uterus, and the sooner these are removed the better.

Later on at the end of a week or 10 days the patient may suffer from a secondary post-partum haemorrhage, and this can cause bleeding of severe degree. On several occasions one of us (G. G. L.) has visited such cases with the Flying Squad and been assured that the placenta and membranes were complete. On investigation quite a large piece of placenta was removed by digital curettage in hospital after resuscitation of the patient. A retained succenturiate lobe may be responsible for this.

\section{Care of the Perineum}

A third-degree tear (involvement of the sphincter ani) should indicate hospital admission for proper repair, but if repair has been undertaken at home it will be necessary for the patient to have her bowel action suppressed for five days. This can

\section{TO-DAY'S DRUGS}

\section{Carbenoxolone Sodium}

This drug is produced by Biorex Laboratories under the name of "biogastrone."

\section{Chemistry}

Carbenoxolone sodium is prepared from liquorice root, which is one of the oldest remedies known to man. Liquorice contains glycyrrhizic acid (glycyrrhizin), which is probably the sweetest natural substance known. From this is prepared the triterpenoid glycyrrhetinic acid, which has a structure similar to that of the steroid hormones of the adrenal cortex. The disodium salt of the 3-0-hydrogen succinate of glycyrrhetinic acid (carbenoxolone) is freely soluble in water.

\section{Pharmacology ${ }^{2}$}

Glycyrrhetinic acid has some steroid-like action in affecting water and electrolyte metabolism. It may cause the retention of water and sodium and chloride ions in the body. It also has be done only by her taking a non-residue diet. On the fourth day an olive-oil enema should be given and a mild laxative such as "senokot" prescribed. (There is no evidence that "senokot" affects the baby through the mother's milk.) Hacmorrhoids are a frequent complication of the puerperium and may result from the patient having been given castor oil, often in repeated doses, in order to get labour to start. The use of castor oil for induction of labour should be given up, since it does nothing to help labour to start and only causes haemorrhoids in the puerperium. Should haemorrhoids still occur, however, they should be treated by astringent suppositories and local anaesthetic ointment. The thrombotic haemorrhoid should be evacuated.

\section{Phlebothrombosis and Pulmonary Embolism}

The patient may develop pain in the chest and slight bloodstained sputum, and signs of collapse at the bases of the chest. These may occur at any time, without her having any prior complaints referable to thrombosis of the veins in her legs. In such a case it is important to get a consultant to see the patient at once. Where there has been no sign of pulmonary embolism and there is marked phlebothrombosis of the legs accompanied by pyrexia and tenderness (positive Homan's sign) the leg should be immobilized immediately and the patient put on heparin 10,000 units intramuscularly six-hourly and "dindevan" (phenindione). The prothrombin time will have to be estimated daily and will have to be kept on a level of two and a half times normal. On this treatment she will be allowed up after 48 hours unless complicated by further pulmonary embolism. "Dindevan" will be continued for at least 14 days.

Thanks are recorded to Dr. R. E. Hemphill, Lecturer in Psychiatry, for his contribution on "Psychiatric Illness in the Puerperium," and Dr. D. W. Barritt for his comments on phlebothrombosis. ${ }^{1}$

\section{REFERENCB}

'Barritt, D. W., and Jordan, S. C., Lancet, 1960, 1, 1309. an anti-inflammatory sction resembling that of hydrocortisone, and some antitussive activity. On the other hand, while hydrocortisone is ulcerogenic, carbenoxolone has an anti-ulcer activity. To what degree liquorice extracts exert any true anti-ulcer activity is still under investigation, but it appears that the active principle causing such effect is in fact carbenoxolone.

\section{Clinical Uses ${ }^{3}$ \& 5}

Carbenoxolone is available as 50-mg. tablets which are taken orally and used in the treatment of gastric ulcer. The recommended dosage is $150-300 \mathrm{mg}$. daily, in the form of one to two tablets three times a day after meals. The action appears to be at least partly a local anti-inflammatory one upon the ulcer, so that the drug is not recommended, as at present formulated, for the trearment of duodenal ulcer. It is recommended as an aid to healing to be given with an unrestricted diet (except for general advice usual in cases of peptic ulcer) and with no special limitations as regards normal activities. The usual course of treatment lasts from three to four weeks, 